\title{
COVID-19 Infection in India: Seropositivity versus the Dynamics of the Spread
}

\author{
Zameer Shervani, Deepali Bhardwaj, and Roma Nikhat
}

\section{ABSTRACT}

\begin{abstract}
The self-referred serosurvey data showed that the whole of India, 31\% population has SARS-CoV-2 antibodies. Seroprevalence was higher in females (35\%) than males (30\%). The seropositivity in Ahmedabad, Jaipur, Bangalore, and Delhi increased monotonously from July to December. Seropositivity in big cities of India was as high as $63.8 \%$ in Bangalore followed by Delhi $(\mathbf{5 4 . 9 \%})$ and Kolkata (54.6\%). In cities (Mumbai, Chennai, Pune, Coimbatore, Surat, Visakhapatnam, Kolkata, and Nagpur) antibodies prevalence first increased with time after a threshold it started decreasing in 4 month time in most of the cities. Intergeneration age dependent seropositivity for both males and females showed an "M-shaped" pattern. The seroprevalence versus monthly infection rate in Delhi and Kolkata showed an increase in seroprevalence with the caseload. In Chennai, the caseload of new infections decreased while seropositivity increased. In the case of Mumbai, seropositivity increased with increase in the caseload in the beginning till October, after that with decrease in the caseload seroprevalence also diminished. In none of the cities, the amount of antibodies titers was enough to stop the second wave of the pandemic.
\end{abstract}

Keywords: COVID-19 serosurvey, First and second COVID-19 waves, Herd immunity, Seroprevalence in India, SARS-CoV-2 spread.
Submitted : June 27, 2021

Published : July 31, 2021

ISSN: $2593-8339$

DOI: $10.24018 /$ ejmed.2021.3.4.953

\section{Zameer Shervani*}

Food \& Energy Security Research \& Product Centre, Sendai, Japan.

(e-mail: shervani.nanotek@gmail.com) Deepali Bhardwaj

Centre for Skin and Hair Pvt. Ltd. D 305, Defence Colony, First Floor, New Delhi, India.

Roma Nikhat

Food \& Energy Security Research \& Product Centre, Sendai, Japan.

*Corresponding Author

\section{INTRODUCTION}

The COVID-19 pandemic is not yet under control. The SARS-CoV-2 virus is appearing repeatedly in the forms of variants and waves. Furthermore, the immunization coverage is far from over leaving the populations unprotected. The new delta variant of COVID-19 virus is highly transmissible, first identified in India, taking hold [1] of Europe making difficult to ease restrictions. The variant can evade some vaccines. Due to the recent surge [2] of coronavirus cases, Japan has declared a state of emergency until May 31, 2021, in three more prefectures Hokkaido, Okayama, and Hiroshima adding to the list of prefectures already in an emergency. About $70 \%$ of Japan's population under restricted activities like 8 P.M. closing hours for eateries and no alcohol served in bars and restaurants. Also, Japan has to prepare for hosting the Olympics beginning from July 23. The state of emergency will cost Japan \$ 9.1 billion loss from gross domestic product and 57,000 job losses. Japan's vaccination drive is slowest among group seven (G7) nations, only 3\% population has received the shots. However, the situation in Japan has improved recently. In the fourth wave [3] of infection, on June 14 the lowest number of 938 new coronavirus case were reported [4]. The hard hit in the fourth wave, Osaka Prefecture reported 57 new coronavirus cases, the lowest in three months while Tokyo confirmed 209 new cases. In a new development on the inoculation front, Japan Airlines Co. begins vaccination for employees in workplace inoculation program. A day before, All Nippon Airways Co. was the first to start this program. A similar workplace vaccination program is expected to be taken by other agencies. Universities in Japan may start in-campus vaccination to speed up the country`s inoculation drive.

The COVID-19 pandemic situation in the second most populous country India became worse in the second wave of infection. As of June 13, India recorded 67,699 new cases and 3,863 deaths taking the total tally to $29,512,862$ cases and 371,440 deaths. Three states Tamil Nadu, Kerala, and Maharashtra contributed most to India`s caseload on June 13. Tamil Nadu, Kerala, and Maharashtra reported 14,016; 11,584 and 10,442 new cases, respectively. The fatalities were 2,771; 267, and 206 in Maharashtra, Tamil Nadu, and Kerala, respectively. In the case of Maharashtra, the deaths are higher due to the missed backlog cases. Till June 13, only $15 \%$ of India`s population has received at least one vaccine shot while $3.5 \%$ of the population has been vaccinated fully. The second wave of infection in India might be receding but India still recorded the highest 90,090 daily new cases in the world on June 12. The other two countries Brazil and Colombia were in second and third places with 66,770 and 25,384 cases, respectively. As of June 12, India`s share of the world's coronavirus caseload was at a high of $25 \%$ [5]. Maharashtra is one of the worst affected states. In the first COVID-19 wave which was for 11 months (March 9, 2020- 
February 9, 2021), Maharashtra had 2,048,802 cases that caused 51,360 fatalities [6]. The second wave in Maharashtra which began on February 10 and till now in four months has caused 3,868,319 new infections and 61,336 fatalities. The deaths and coronavirus cases in the short (4 months) second wave are huge than the longer (11 months) first wave. The first wave was milder than the severe second wave. India must be ready to face the third wave.

The SARS-CoV-2 herd immunity concept was first tested and established [7] in Dharavi Slums (Mumbai, India). The serosurvey data of Dharavi and Cuffe Parade slums of Mumbai (India) were analyzed. The first and second serosurvey of Dharavi showed the seroprevalence of 57 and $45 \%$, respectively while the Cuffe Parade Mumbai slums have $75 \%$ seroprevalence in population as determined in the survey of October 2020. If a survey is conducted in Dharavi slums it will also show seropositivity of $75 \%$ the same as recorded in Cuffe Parade slums. The absence of the second peak and no new coronavirus cases recorded on several occasions prove that the Dharavi slums have achieved herd or mass immunity without vaccinating the population. The higher infection rate in the state of Kerala (India) compared to other Indian states was explained first by the newly established "Health Index Theory" [8], [9]. The viability, infectious nature, and sanitization of novel coronavirus were described in a recently published article [10].

The article [11] described the possibility of the SARSCoV-2 virus transmission from infected humans to animals and pets. The development in COVID-19 detection, treatment, and vaccine preparation has been explained separately [12]-[14]. In this review article, the relationship between seroprevalence in 12 most populated cities of India and SARS-CoV-2 infection rate have been studied. The data of self-referred serosurvey conducted [15] in urban India have been considered. The purpose of the research is to know how much the population has been exposed to the virus. The study is very useful for the vaccination strategy in India and other parts of the globe.

\section{METHOD}

The article [15] contains the details of the method used to conduct the serosurvey and collect the data. In brief, Thyrocare Laboratories had IgG antibodies testing facility were employed. The research was supported by the Canadian Institutes of Health Research and University of Toronto, Canada. A total of 448,518 people took the antibodies testing for COVID-19 virus in the period June 2020-December 2020. The data of SARS-CoV-2 infection were obtained from the source mentioned [16]. Since the antibodies testing costed money, the survey covered only the wealthy population. The seropositivity data given in this article do not represent the uniform random urban population. In real on the ground seroprevalence in urban India has not been reported yet. From the recorded seropositivity, we have estimated broadly how big the urban population has been infected so far.

\section{RESULTS AND DISCUSSION}

\section{A. Serosurvey Results of 3 Mega and 9 Large Cities of India}

Fig. 1 (a) is the seropositivity (\%) versus date (months the survey conducted) plots for cities Bangalore, Jaipur, Ahmedabad, and Delhi for July-December 2020. In July, the SARS-CoV-2 seropositivity in the cities of Ahmedabad, Jaipur, Bangalore, and Delhi was 11.1, 13.0, 14.0, and 25.2\%, respectively. The seroprevalence in the population kept increasing monotonously. By December, seropositivity reached 48.4, 63.8, 42.3, and 54.9\% in the cities Ahmedabad, Jaipur, Bangalore, and Delhi, respectively. The increase in seroprevalence was highest in Jaipur. The seroprevalence in the cities Mumbai, Chennai, Pune, and Coimbatore are shown in Fig. 1 (b) while the seroprevalence in Surat, Visakhapatnam, Kolkata, and Nagpur is shown in Fig. 1 (c). Unlike Fig. 1 (a) where seroprevalence increased with the passing months (July to December), Fig. $1 \mathrm{~b}$ and c showed that the seropositivity for the cities mentioned in the figures first increased after reaching a maximum the seroprevalence decreased.

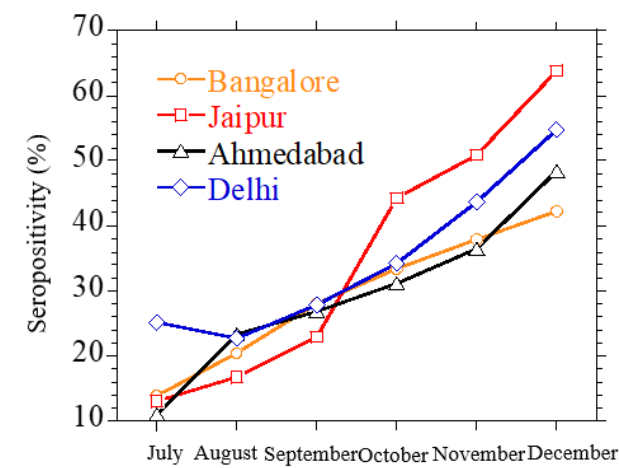

Fig. 1 (a). SARS-CoV-2 seropositivity (\%) in Bangalore, Ahmedabad, Jaipur, Delhi.

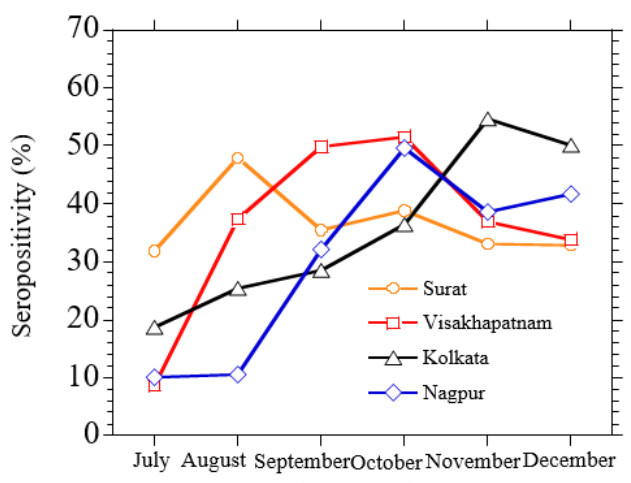

Fig. 1 (b). SARS-CoV-2 seropositivity (\%) in Surat,Visakhapatnam, Kolkata, Nagpur.

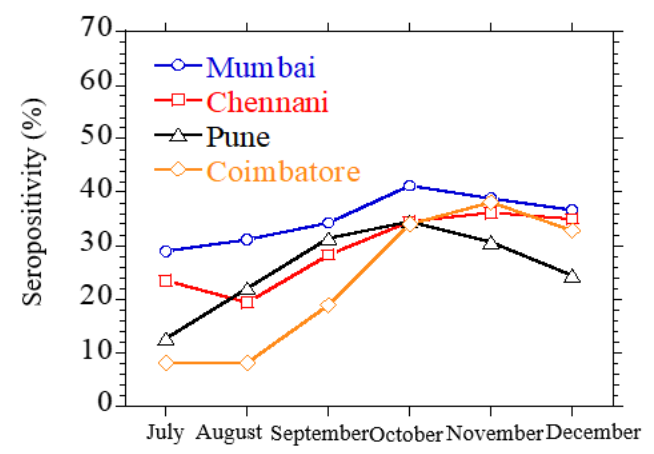

Fig. 1 (c). SARS-CoV-2 seropositivity (\%) in Mumbai, Chennai, Pune, Coimbatore. 
The cities mentioned in Fig. 1 (b) and (c) can be divided into two groups. The seropositivity maximum in Fig. 1 (b) is in the range of $34-41.3 \%$ and Fig. 1 (c) of $47.9-54.6 \%$. The maximum seroprevalence is in the order of $63.8,54.9$, and $54.6 \%$ in the city of Jaipur (Fig. 1 (a)), Delhi (Fig. 1 (a)), and Kolkata (Fig. 1 (b)). In Figures 1a and b, the sereprevalence started decreasing in most of the cities after 4 months. The decrease in the seroprevelance in the population is a matter of concern for developing the mass or herd immunity in the population. But some experts say that the antibodies formed from previous coronavirus infections may give protection against reinfections no matter antibodies titers are diminished over time. The waning out of antibodies does not translate into a lack of immunity against the virus. There are "memory cells" that can recall the SARS-CoV-2 pathogen to put a defense against the virus [17]. The seroprevalence in 3 mega cities (Mumbai, Delhi, and Kolkata), 9 big cities (Surat, Visakhapatnam, Nagpur, Ahmedabad, Jaipur, Bangalore, Chennai, Pune, and Coimbatore), and all of India are shown in Fig. 2. The seropositivity in mega cities was higher $(33.3 \%)$ than in big cities $(27.3 \%)$. In mega cities there are more crowded places and closed spaces and increased contacts than in big cities are the reasons for higher positivity. In terms of gender, the seropositivity is higher in females (34.6\%) than in males (29.9\%) (Fig. 3). A similar higher seroprevalence was found [7] in women than men in Mumbai slums of Dharavi and Cuffe Parade.

Age distribution of seropositivity has been shown in Fig. 4. The seroprevalence in the age wise population $<20,20-44$, $45-69$, and $\geqq 70$ years was $32.0,27.8,37.5$ and $39.1 \%$, respectively. Fig. 5 is the age and gender-specific seroprevalence. In both male and female population " $\mathrm{M}$ shaped" pattern was recorded which was the same as normally noticed in intergeneration virus spread. The prominent features of the plots are that in the age group 5-9 and 10-14 years the seroprevalence in males and females is nearly the same while $>14$ years women have more antibodies than men. At the age of 70-74 and $80-80$ plus, the trend reversed with men having more antibodies than women.

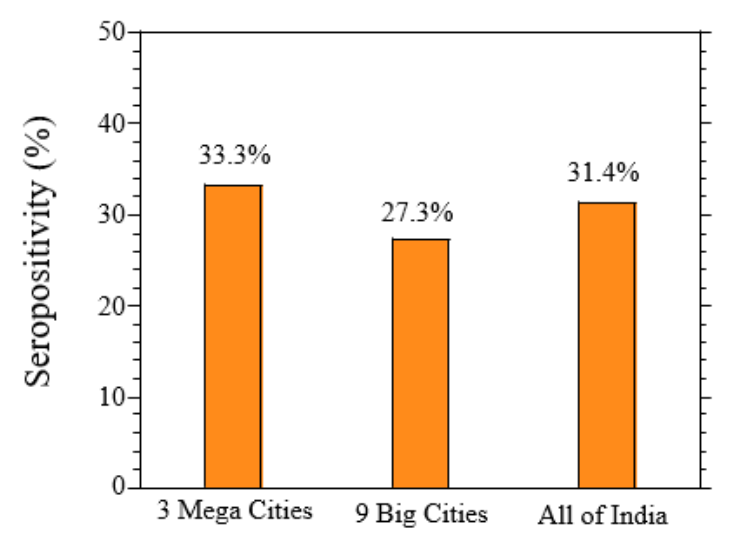

Fig. 2. Seropositivity to SARS-CoV-2 in 3 mega cities Mumbai, Delhi, Kolkata), 9 big cities (Chennai, Bangalore, Ahmedabad, Pune, Surat,

Jaipur, Nagpur, Coimbatore, Visakhapatnam), and All of India. Survey was for the months June-December 2020.

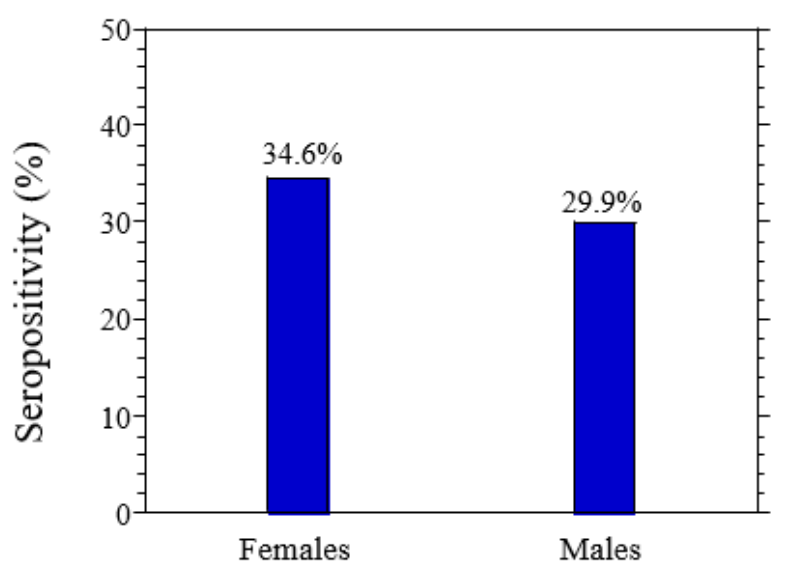

Fig. 3. Seropositivity to SARS-CoV-2 in females and males in all of India for the period June-December 2020.

Survey was for the months June-December 2020.

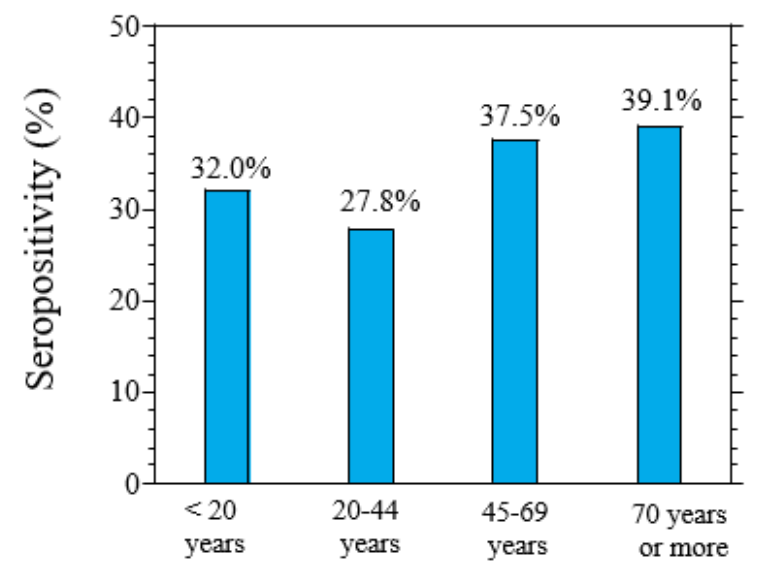

Fig. 4. Age distribution of seropositivity to SARS-CoV-2 in all of India for the period June-December 2020.

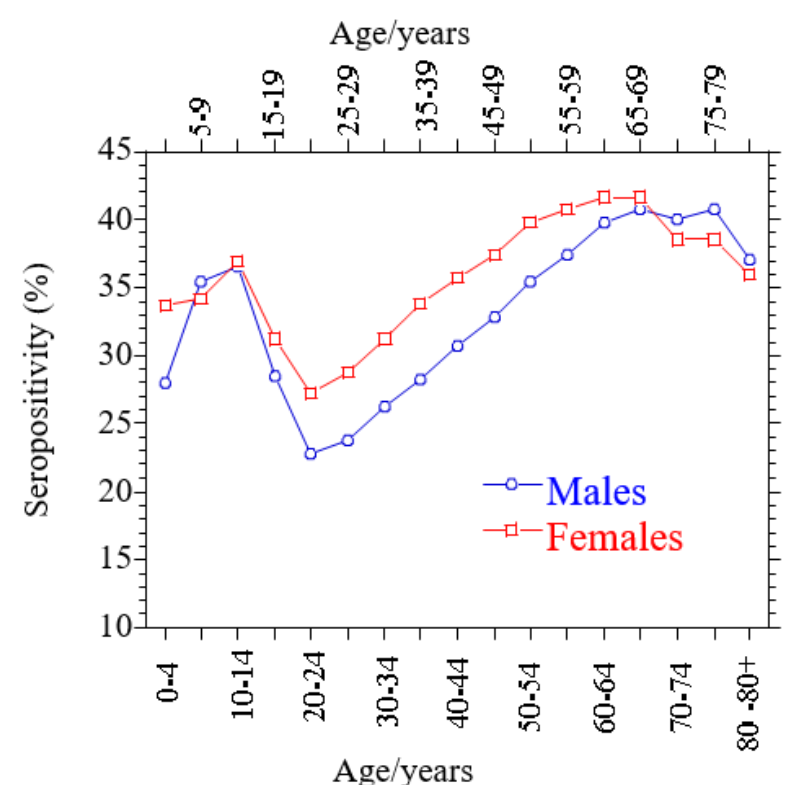

Fig. 5. SARS-CoV-2 seroprevalance (\%) distribution by age and sex for July-December 2020 in India.

\section{B. Seropositivity versus Infection Rate in Big Cities of India}

Fig. 6 shows the plots of seropositivity (\%) and caseload (per million) of Delhi city recorded in the months shown in the figure. Seroprevalence increased monotonously from the month of August to December 2020. Seroprevalence in 
August was $22.8 \%$ and reached a value of $54.9 \%$ by December. The number of the new coronavirus caseload also increased from August to November 2020, monotonously. The immunity that developed in the $54.9 \%$ population of Delhi was not enough to stop the surge in the cases that began in March 2021 and continued till April. The seropositivity and monthly caseload for Mumbai city has been shown in Fig. 7. Compared to July, new daily cases in August decreased. Again, in September cases started increasing. The cases are fewer in October compared to September. After September cases started decreasing. In the year 2021, cases surge to a larger extent from February onward and continued till April. In May there was a decrease again in the second wave of the pandemic. Seroprevalence increased from the month July and kept increasing till October, reached a value of $41.3 \%$ and then started decreasing. The prevalence of antibodies in the $41.3 \%$ of the population in Mumbai could not stop the second wave of the pandemic though.

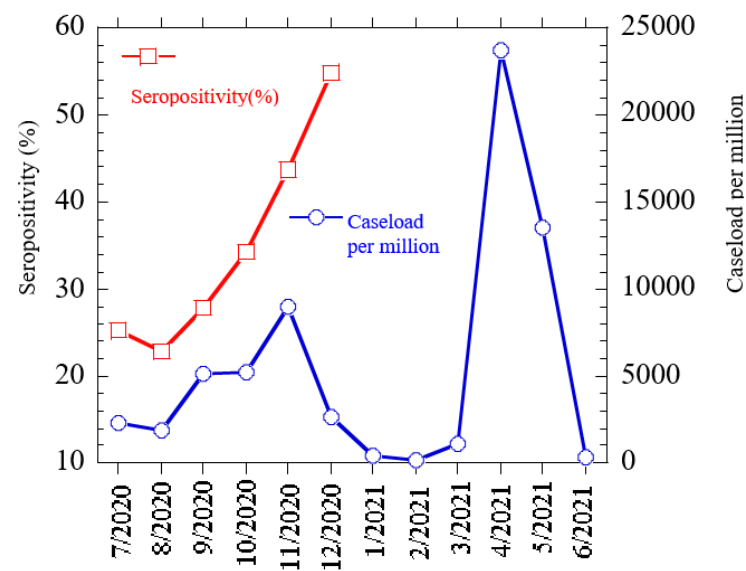

Fig. 6. Comparison of seropositivity and COVID-19 caseload in Delhi.

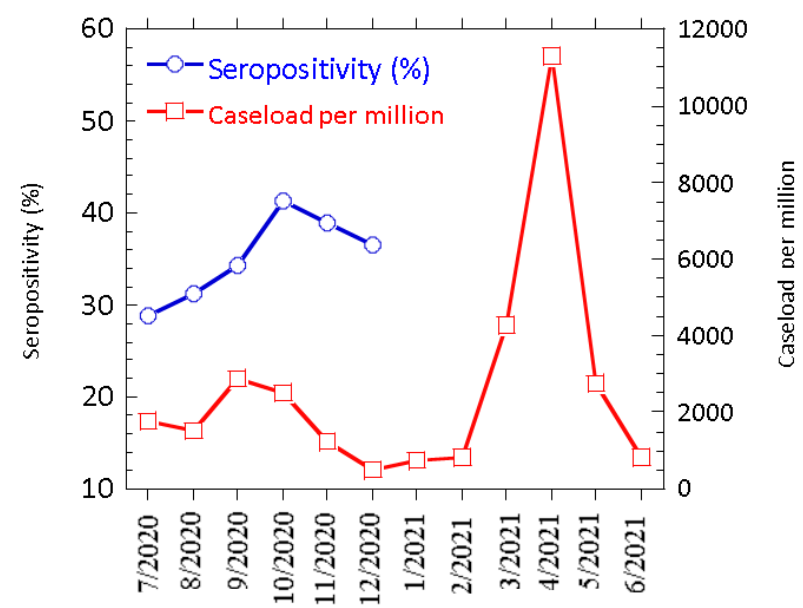

Fig. 7. Comparison of seropositivity and COVID-19 caseload in Mumbai.

Fig. 8 is the seroprevalence and monthly caseload recorded in Kolkata. The number of new cases increased in August compared to July. In September there was a decrease, in October cases started increasing. In December, cases decreased from the previous month of November. After decreasing till February, cases started increasing again from February onward and a big surge in the cases was noticed in April and continued till May. Seroprevalence increased from July and increase continued till November, however, it decreased in December. The seroprevalence in $54.6 \%$ population of Kolkata did not stop the surge in the cases in March 2021. In Chennai city, from July cases started decreasing (Fig. 9) and decline continued till February 2021. In March a big surge in infection was recorded and a spurt in the cases continued till May 2021. Seroprevalence in Chennai population increased from August onward and continued increasing till November 2020. In December a slight dip in seropositivity was recorded. Maximum seroprevalence $(36.2 \%)$ recorded in November was not enough to stop the second wave.

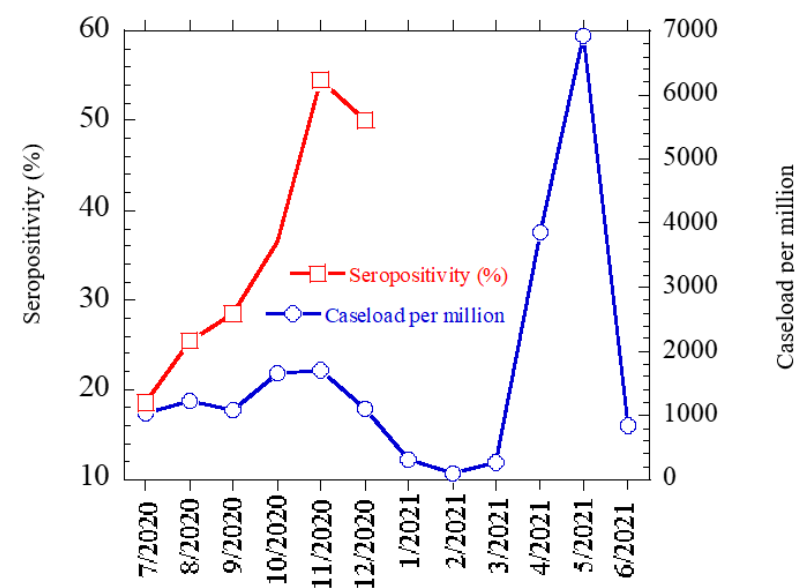

Fig. 8. Comparison of seropositivity and COVID-19 caseload in Kolkata.

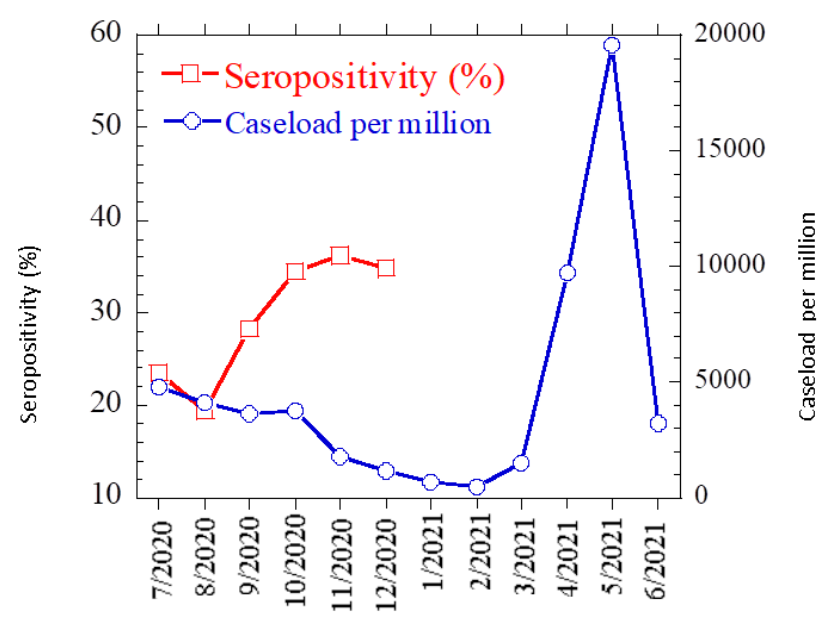

Fig. 9. Comparison of seropositivity and COVID-19 caseload in Chennai.

\section{STATEMENTS}

The data and results in this article are very reproducible. Author (Zameer Shervani, Ph.D.) is Director of Food \& Energy Security Research \& Product Center, Sendai, Japan. Authors have qualifications; Deepali Bhardwaj MBBS, MD, DVDL, M.Phil.; Roma Nikhat MCA, MBA. Correspondence detail: WhatsApp: +818033214889 .

\section{REFERENCES}

[1] Delta variant is taking hold of Europe: WHO, The Hindu, June 10, 2021.

[2] Japan widens virus emergency to three more prefectures, Reuters, May 14, 2021.

[3] Fear of '4th wave' rise with surging rural Japan coronavirus infections, The Mainichi, March 26, 2021. 
[4] Japan daily COVID-19 cases below 1,000 for the first time since March, The Japan Times, June 14, 2021.

[5] Only 3.5\% of the population is fully vaccinated as of June 13, 2021, The Hindu, June 13, 2021.

[6] Covid second wave: $3.8 \mathrm{mn}$ new cases, $61 \mathrm{~K}$ deaths in 4 months in Maharashtra, Hindustan Times, June 15, 2021.

[7] Shervani et al. Dharavi Slums (Mumbai, India): The Petri Dish of COVID-19 Herd Immunity, European Journal of Medical and Health Sciences, 3, 2021, 38-41.

[8] Shervani Z, "COVID-19 in Kerala: Health Index Theory," European Journal of Medical and Health Sciences, 3, 2021, 21-24.

[9] Shervani Z, "COVID-19 in Kerala: The Dynamics of Spread and Health Index Theory," RAS Medical Science, 2021, 1, 1-3.

[10] Shervani Z, et al. "Viability of SARS-CoV-2 and Sanitization Methods," European Journal of Medical and Health Sciences, 3(2021)22-27.
[11] Shervani Z, et al. "Risk of SARS-CoV-2 Transmission from Humans to Pets and Vice Versa," European Journal of Medical and Health Sciences, 3(2021)34-38.

[12] Shervani Z, et al. "SARS-CoV-2 Delayed Tokyo 2020 Olympics: Very Recent Advances in COVID-19 Detection, Treatment, and Vaccine Development Useful Conducting the Games in 2021," Advances in Infectious Diseases, 2020; 10: 56-66.

[13] Shervani Z, et al., "COVID-19 Vaccine," Advances in Infectious Diseases, 2020;10: 195-210.

[14] Shervani Z, et al., "World's Fastest Supercomputer Picks COVID-19 Drug," Advances in Infectious Diseases, 2020; 10: 211-225.

[15] Velumani A., et al. SARS-CoV-2 Seroprevalence in 12 Cities of India from July-December 2020, doi: https://doi.org/10.1101/2021.03.19.21253429.

[16] COVID19India.org.

[17] Covid-19 antibodies diminish over time, but experts say there's no reason to be alarmed, NBC NEWS.COM, October 31, 2020. 